\title{
Heterozygous app a7 I 3 t mutation carrier with inflammatoy amyloid angiopathy and family history of alzheimer's disease. First case in Argentina
}

\begin{abstract}
Aim: To report the case of a patient who suffered from cerebral amyloid angiopathy due to an autosomal dominant mutation in the APP gene

Design/Methods: Medical record and neuroimaging revision. DNA extraction from the saliva sample. Sanger sequencing of the coding regions of the following: APP (NCBI RefSeq NM_000484.3), PSEN1 (NCBI RefSeq NM_000021.3) y PSEN2 (NCBI RefSeq NM_000447.2)

Clinical case: A male patient of 71-year-old with a past medical history of recurrent lobar hemorrhagic strokes leading to major cognitive decline since the age of 65 , prominent cerebral microangiopathy was present and worsened progressively. Patient's mother had presenile Alzheimer's disease. The patient developed partial nonconvulsive status epilepticus, no evidence of new strokes (ischemic or hemorrhagic) was found. Two weeks after seizures a new MRI unveiled right frontal meningeal enhancement. Lumbar puncture and cultures were normal. Inflammatory amyloid angiopathy was suspected. A course of IV methylprednisolone was administered followed by oral steroids with a slight improvement. The patient died four months after due to clinical complications. Post-mortem analysis confirmed a heterozygous mutation: c. $2137 \mathrm{G}>\mathrm{A}$; p. $\operatorname{Arg} 713 \mathrm{Thr}$ at exon 17 of the APP gene.

Conclusion: The A713T mutation has been reported by groups of European researchers (British, Spanish and Italian) with variable phenotypes. This would be the first case detected in Argentina to our knowledge. It is notable the main manifestation in our case was the presence of recurrent hemorrhagic stroke, however, the antecedent of a pre-senile AD in a first-degree relative suggested the presence of genetic etiology. This mutation may be underdiagnosed. It would be advisable that a complete genogram must be performed in patients with cerebral amyloid angiopathy. The detection of these cases has implications for genetic counselling.
\end{abstract}

Keywords: familiar alzheimer disease, amyloid angiopathy, APP, A713T
Volume 9 Issue 2 - 2019

\author{
Marcos C Fernández Suarez, ${ }^{1,4}$ Ignacio \\ Brusco, ${ }^{3}$ Carolina Damasso, ${ }^{2}$ Natividad \\ Olivar, ${ }^{3}$ Laura Morelli, ${ }^{2}$ Griselda Russo ${ }^{1,4}$ \\ 'Neuropsiquiatras Center, Argentina \\ 2Leloir Institute -IIBBA-CONICET,Argentina \\ ${ }^{3}$ INEAR-Alzheimer Argentina, Argentina \\ ${ }^{4}$ Fundacion de Neurociencias del Alto Urugual (FUNAU), \\ Argentina
}

Correspondence: Marcos Fernández Suarez, Neuropsiquiatras Center, Forest Ave II 79 5C, Cl42 I CEI, Buenos Aires, Argentina, Email fernandezsuarezmarcos@gmail.com

Received: April 01, 2019 | Published: April 09, 2019
Abbreviations: AD, alzheimer's disease; APP, amyloid precursor protein gene; CAA, cerebral amyloid angiopathy; EOAD, early onset alzheimer's disease; LOAD, late onset Alzheimer's disease; PSEN1, presenilin 1 gene; PSEN2, presenilin 2 gene

\section{Introduction}

Familial presentation of Alzheimer disease (AD) accounts for approximately $5 \%-10 \%$ of all AD cases. Three genes: the amyloid precursor protein (APP), presenilin 1 (PSEN1), and presenilin 2 (PSEN2), were reported as associated with an early-onset form of the disease (EOAD). Several mutations (130 mutations) have been described in PSEN1, 8 in the PSEN2 gene and about 20 in APP (available at http://molgen-www.uia.ac.be/ADmutations). ${ }^{1,2}$ In regards APP mutations, many are considered pathogenic, but other are polymorphisms or silent mutations. The A713T mutation is associated with a variable phenotype. Some carriers exhibit clinical and neuropathological features typical of Alzheimer's disease, while others have a more complex presentation, with prominent cerebrovascular disease and cerebral amyloid angiopathy (CAA).
Asymptomatic carriers have also been documented. We described the case of a 71-year-old man who presented recurrent lobar hemorrhagic strokes (a chronic form of CAA) with suspected inflammatory response; this patient was a carrier of a heterozygous APP A713T mutation and had family history of EOAD.

\section{Case presentation}

A 71-year-old man was assessed in 2016 at the emergency department due to alert deterioration caused by dehydration, his past medical history was remarkable. He had suffered from recurrent lobar hemorrhagic stroke since age 65. 2009 (right frontal), 2013 (left temporal) (Image 1), leading to an amyloid angiopathy diagnosis base on Boston Criteria; during 6 months in 2012 a low dose of oral prednisone was administered due to a suspicion of inflammatory amyloid angiopathy. After fluid administration the patient recovered to his baseline status: a major cognitive deterioration, left hemiparesis and severe mix aphasia was observed as sequelae, he was able to walk supervised. His relatives reported a gradual cognitive deterioration during the past year. 

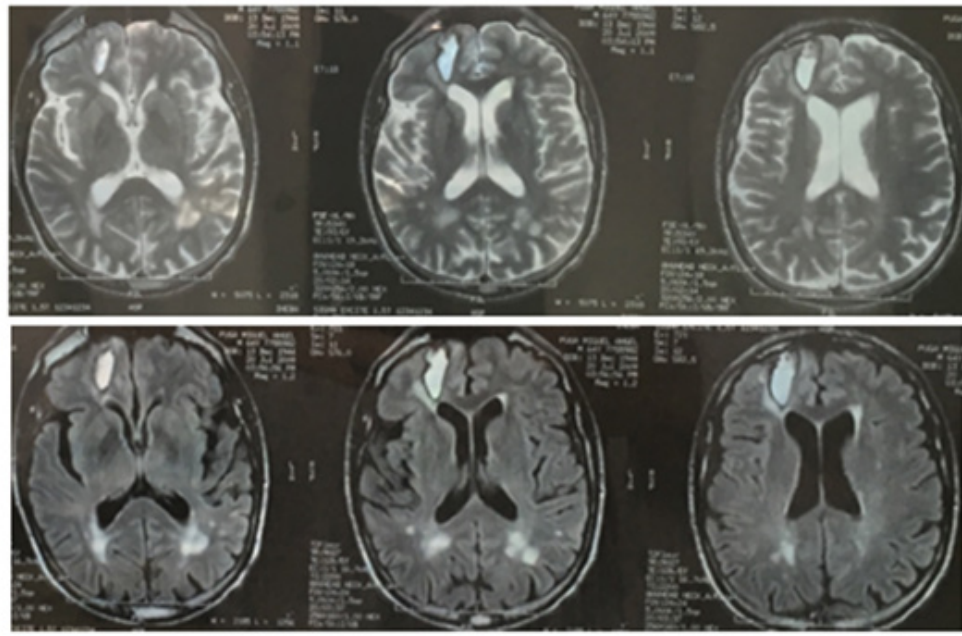

A)

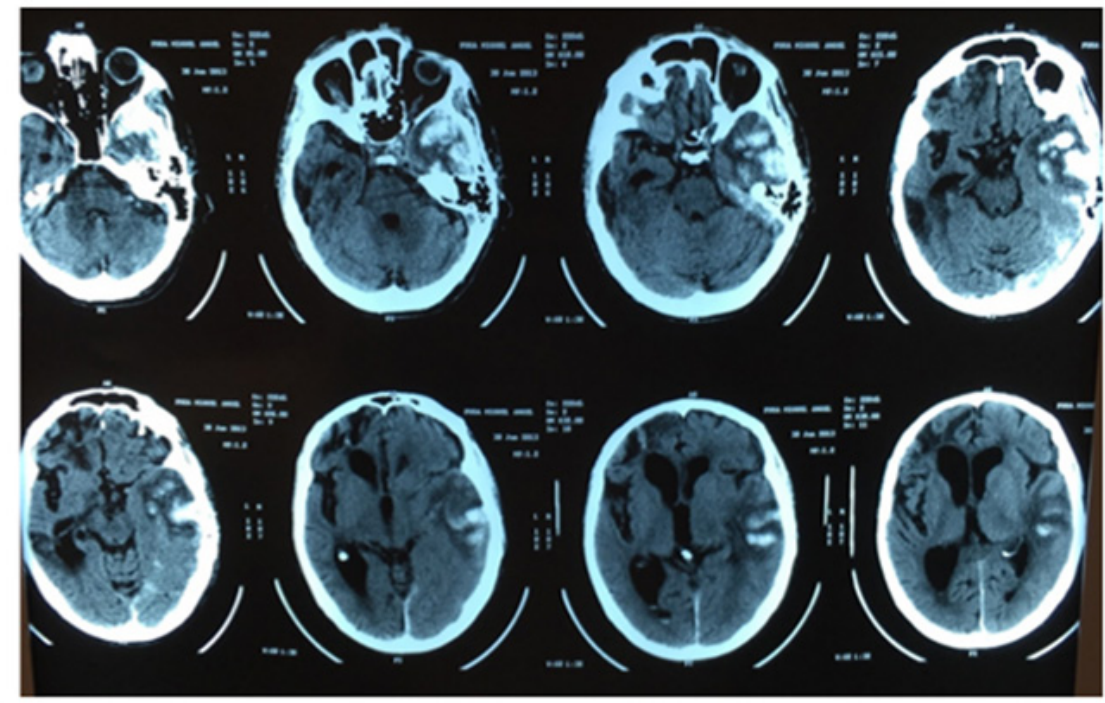

B)

Image I A) Axial MRIT2 and FLAIR. Right frontal hematoma. Subcortical white matter lesion (2009). B) CT Scan. Axial. Left temporal hematoma. (20I3)

Familiar antecedent of EOAD (patient's mother) without clinical cerebrovascular disease was reported by relatives. One older brother and two siblings were healthy. Four months after the initial assessment a tapering of valproic acid was initiated, because of he had none record of seizures and was receiving a suboptimal dosing of $500 \mathrm{mg} /$ day. The patient developed a status epilepticus, starting as partial left motor clonic movements, followed by a generalized tonic-clonic seizure. After IV Levetiracetam's infusion clinical seizures stopped but EEG evidenced frequent right frontal electrographic seizures. Lacosamide $200 \mathrm{mg}$ oral charge finally stopped seizures.

Despite of EEG normalization, clinical status did not improve. Lumbar puncture was normal, Brain CT and MRI showed old ischemic and hemorrhagic sequelae. After 2 weeks mental status was below his previous baseline, hyperactive and hypoactive delirium persisted despite antipsychotic medication. A new contrast-MRI showed right frontal pachymeningeal, leptomeningeal enhancement, and gyral edema in FLAIR/T2 sequences, the same was retrospectively observed in the first non-contrasted MRI (Image 2). Despite atypical neuroimaging and age, inflammatory amyloid angiopathy was suspected due to meningeal enhancement, new seizures onset and continuous cognitive deterioration during the months before hospitalization. As a differential diagnosis post seizures edema was proposed, but none DWI restriction was observed in the first and second MRI within 2 weeks. Finally, $3 \mathrm{~g}$ of methylprednisolone was administered followed by oral prednisone. A slight improvement was observed (the patient recovered from prostration and start walking again, but persisted markedly confused). 3 months later the patient died from pneumonia. 

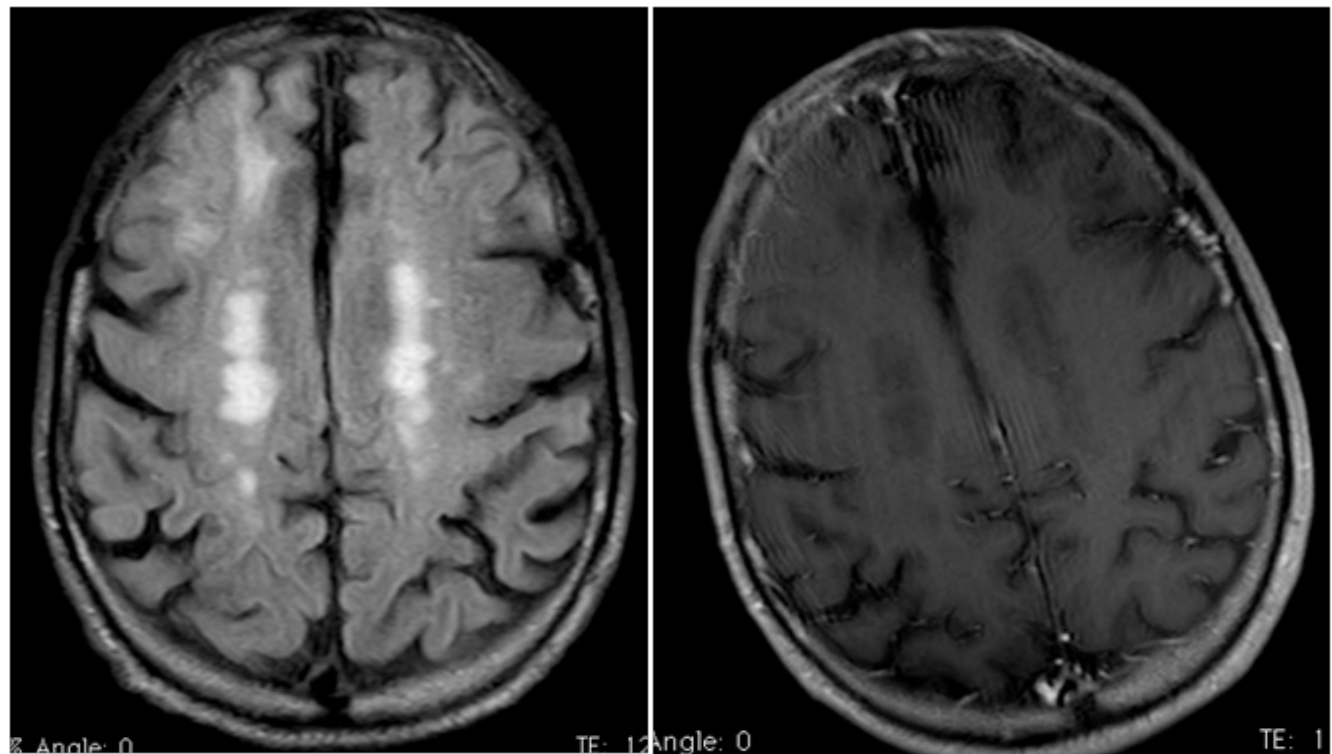

Image 2 Axial FLAIR and AXIALTI gadolinium enhanced, right frontal meningeal.

The familiar antecedents rise suspicion of an amyloid precursor protein (APP) mutation in the kindred and after informed consent PSEN1, PSEN1 and APP gene analysis was performed using Sanger sequencing method. ADN was extracted from a saliva sample. Result from a heterozygous mutation c. $2137 \mathrm{G}>\mathrm{A} ; \mathrm{p}$.Arg $713 \mathrm{Thr}$ in 17 exon of APP gene was received post mortem (Image 3).

\section{\begin{tabular}{lllll}
$\mathrm{V}$ & $\mathrm{I}$ & $\mathrm{T}$ & $\mathrm{T}$ & $\mathrm{V}$ \\
\hline
\end{tabular}}
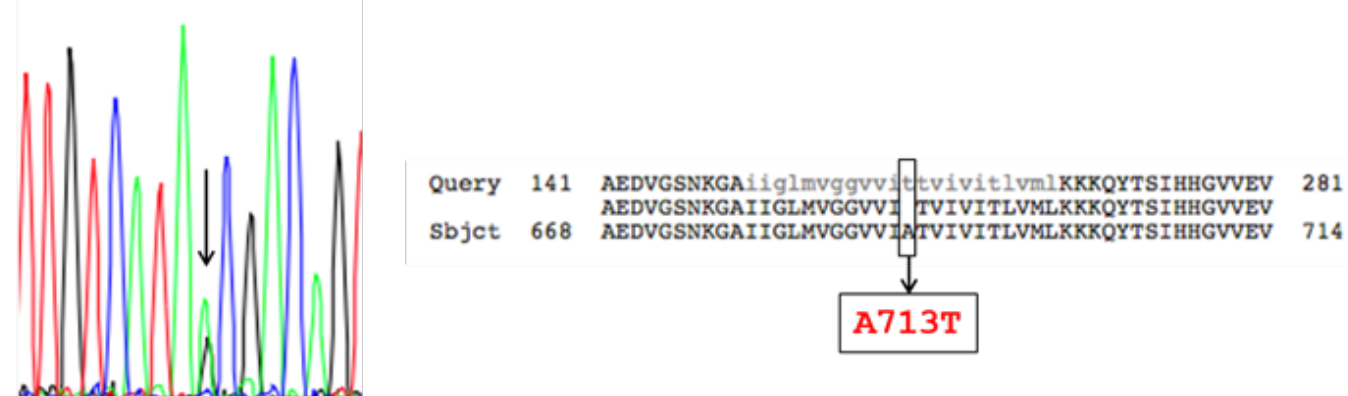

TTGTCATA [A/G] CGACAGTG

$$
5, \cdots-3^{\prime}
$$

Image 3 Genetic testing

\section{Discussion}

The APP A713T mutation has been reported with dominant inheritance and in heterozygosis, associated with familial AD with both early and late onset and cerebrovascular lesions. ${ }^{3}$ Unlike PSEN1 and PSEN2, causative APP mutations are clustered into just 2 exons (16 and 17), both of which are less than 500 base pairs and therefore amenable to screening via Sanger sequencing. ${ }^{4}$

The first report from 1992, described a sample of 130 EOAD patients that were screened for this mutation, finding 1 case of a 64 -year-old female. 5 relatives were asymptomatic carriers, $(2$ of them older than 62 and one of 88 -year-old). No stroke-like manifestation was reported. 123 non-affected individuals were screened too, with negative results. ${ }^{5}$ In 2004 another group ${ }^{3}$ from Italy, described that six affected subjects were identified in a family; the age at onset ranged from 52 to 68 and the age at death from 57 to 73 , in the similar age of our patient. Two prob and developed early onset dementia, stroke like episodes, with white matter lesions without hemorrhages or hemosiderosis. Post mortem and biopsy pathological study showed AD pathology, amyloid angiopathy and infarcts in the territory of the long penetrating arteries. The junction between the cortex and the subcortical white matter and the periventricular white 
matter were mostly involved. Our case displayed a combination of both haemorragic strokes and periventricular/white matter ischemic lesions. Authors considered that penetrance of A713T could not be determined in that study due to the young age of many carriers. In 2004 another group Armstrong et al. ${ }^{6}$ described a case of A713T mutation's carrier of 56 year old with 7 years of cognitive decline which leads to severe dementia and prostration; postmortem analysis showed diffuse atrophy and $\mathrm{AD}$ pathology, CAA restricted only to meningeal and parenchymal vessels.

As in our case it seems that in the same kindred a variety of clinical manifestations are possible, but the most elusive could be the presentation of late onset $\mathrm{AD}$ with brain microangiopathy as described by Bernardi et al. ${ }^{7}$ in 2009. The researchers found A713T mutation in three of $59(5 \%)$ unrelated patients, which age of onset was between 72-82 years. ${ }^{7}$ However, despite de prevalence in their study, at the same time, researchers suggest that probably this mutation is extremely rare in worldwide population, since all the cases had ancestors in a limited area of Italy. A later study from England, based on the analysis of 451 EOAD, detected only 2 carriers of A713T mutation. ${ }^{4}$

The larger description of a kindred was performed by Conidi et al. ${ }^{8}$ over a 6 generations, including homozygotic and heterozygotic carriers. They found no differences in clinical phenotype in those patients, which suffered from late onset dementia with cerebral microangiopathy. According the mentioned articles A713T is present mostly in Europe. Argentina has received a massive European immigration during XX century, mostly from Italy and Spain, it could be possible that our patient had an European ancestor.

Finally, in this paper we highlight the prominence of recurrent hemorrhagic strokes (clinical amyloid angiopathy), and cerebral microangiopathy at a relative young age over the typical Alzheimer's disease phenotype, reported in previous studies. The rapid decline during the last year of disease with seizures, meningeal enhancement and gyral swelling close to an old stroke, suggested a SNC inflammatory response.

This report has many limitations; first, no medical record related to patient's mother was available, the information was based on relative's information, second, neither a brain biopsy or postmortem anatomopathological analysis was performed, it would be relevant to confirm the presence of $\mathrm{AD}$ pathology and the theoretical inflammatory response. Finally, the relatives decline to receive any information related to genetic counselling, closing the possibility to analyze for asymptomatic carriers too. The A713T mutation has been reported by groups of European researchers. They described variable phenotypes (EOAD, LOAD with or without cerebral microangiopathy due to CAA). This would be the first case detected in Argentina to our knowledge. It is notable that the main manifestation in our case was the presence of recurrent hemorrhagic stroke, however, the antecedent of pre-senile $\mathrm{AD}$ in a first-degree relative suggested the presence of genetic etiology. This mutation may be underdiagnosed.

It would be advisable that a complete genogram must to be performed in patients with cerebral amyloid angiopathy and prominent white matter ischemic lesions.

\section{Acknowledgments}

Genetic testing were completed via Leloir Institute, funding: PBIT 09/14 Grant, Directed by Dr. Laura Morelli, Thanks to Guillermo Americo Russo $†$ for the technical helping in the interpretation of the case.

\section{Conflicts of interest}

Authors declare no conflicts of interest.

\section{References}

1. Sassi C, Guerreiro R., Gibbs R., et al. Exome sequencing identifies 2 novel presenilin 1 mutations (p.L166V and p.S230R) in British early-onset Alzheimer's disease. Neurobiol Aging. 2014;35: 2422.e13-e16.

2. Nicolas G, Wallon D, Charbonnier C, et al. 2015. Screening of dementia genes by whole-exome sequencing in early-onset Alzheimer's disease: input and lessons. Eur J Hum Genet. 2016;24(5):710-716.

3. Rossi G, Giaccone G, Maletta R, et al. A family with Alzheimer disease and strokes associated with A713T mutation of the APP gene. Neurology. 2004;63:910-912.

4. Barber IS1, García-Cárdenas JM1, Sakdapanichkul C, et al. Screening exons 16 and 17 of the amyloid precursor protein gene in sporadic earlyonset Alzheimer's disease. Neurobiol Aging. 2016;39:220.e1-7.

5. Carter DA, Desmarais E, Bellis M, et al. More missense in amyloid gene. Nat Genet. 1992;2(4):255-256.

6. Armstrong J, Boada M, Rey MJ, et al. Familial Alzheimer disease associated with A713T mutation in APP. Neurosci Lett. 2004;370(23):241-243.

7. Bernardi L, Geracitano S, Colao R, et al. AbetaPP A713T mutation in late onset Alzheimer's disease with cerebrovascular lesions. J Alzheimers Dis. 2009; 17(2):383-389

8. Conidi ME, Bernardi L, Puccio G. Homozygous carriers of APP A713T mutation in an autosomal dominant Alzheimer disease family. Neurology. 2015;84(22):2266-2273. 